\title{
Comparative Study to Evaluate the Intersystem Association and Reliability Between Standard Pelvic Organ Prolapse Quantification System and Simplified Pelvic Organ Prolapse Scoring System
}

\author{
Raizada Nivedita $\cdot$ Mittal Pratima $\cdot$ \\ Suri Jyotsna $\cdot$ Puri Anurag $\cdot$ Sharma Vivek
}

Received: 19 July 2013/Accepted: 10 March 2014/Published online: 29 March 2014

(C) Federation of Obstetric \& Gynecological Societies of India 2014

\begin{abstract}
Purpose The purpose of this study was to determine the association between the standard pelvic organ prolapse quantification (POPQ) classification system and the simplified pelvic organ prolapse (S-POP) classification system. Method This is an observational study, in which 100 subjects, whose average age was $60 \pm 10$ years, with pelvic floor disorder symptoms underwent two systems of examinations-POPQ classification system and S-POP classification system at Safdarjung hospital—done by four gynecologists (two specialists and two resident doctors) using a prospective randomized study, blinded to each other's findings. Data were compared using appropriate statistics.

Results The weighted Kappa statistics for the intersystem reliability of the S-POP classification system compared with standard POPQ classification system were 0.82 for the overall stage: 0.83 and 0.86 for the anterior and posterior vaginal walls respectively; 0.81 for the apex/vaginal cuff;
\end{abstract}

Raizada N., Senior Resident · Mittal P., Professor ·

Suri J., Senior Specialist

Department of Obstetrics \& Gynaecology, Vardhaman Mahavir Medical College (VMMC) \& Safdarjung Hospital,

New Delhi 110029, India

Puri A., Post Doctoral Trainee · Sharma V. ( $\square)$, Resident Institute of Post Graduate Medical Education \& Research (IPGMER) \& SSKM Hospital, 244, AJC Bose Road, Kolkata 700020, India e-mail: viveksharma123@outlook.com and 0.89 for the cervix. All these results demonstrate significant agreement between the two systems.

Conclusion There is almost perfect intersystem agreement between the S-POP classification system and the standard POPQ classification system in respect of the overall stage as well as each point within the same system.

Keywords Pelvic organ prolapse .

Pelvic organ prolapse quantification (POPQ) .

Simplified pelvic organ prolapse (S-POP) · Hymen .

Examination · Valsalva

\section{Introduction}

Pelvic organ prolapse (POP) is a bulge or protrusion of pelvic organs and their associated vaginal segments into or through the vagina. POP culminates from infirmity of the supportive structures, either by actual tears or by neuromuscular dysfunction, or both. Support of the vaginal canal is provided by the enveloping endopelvic connective tissue and its condensations at the vaginal apex, which form the cardinal uterosacral ligament complex. The endopelvic connective tissue is the first line of support buttressed intimately with the pelvic diaphragm, composed of the levator ani and coccygeus muscles [1]. These muscles provide a supportive diaphragm through which the urethra, vagina, and rectum egress.

The ICS Pelvic Organ Prolapse Quantification (POPQ) examination defines prolapse by measuring the descent of 
specific segments of the reproductive tract during valsalva strain relative to a fixed point, the hymen. The POPQ system describes the anatomic findings of POP without consideration for symptoms and bother perceived by the patient. The system identifies nine locations in the vagina and vulva in centimeters [2]. Although POPQ system is probably more detailed than necessary for general practice, clinicians should be familiar with the POPQ system because most published studies use it to describe research results. Its two most important advantages over previous grading systems are that (i) it allows for the use of a standardized technique with quantitative measurements at straining relative to a constant reference point (i.e., the hymen); and (ii) its ability to assess prolapse at multiple vaginal sites.

The classification uses six points along the vagina (two points each on the anterior, middle, and posterior compartments) measured in relation to the hymen. The anatomic position of the six defined points should be measured in centimeters proximal to the hymen (negative number) or distal to the hymen (positive number), with the plane of the hymen representing zero. Three other measurements in the POPQ examination include the genital hiatus, perineal body, and the total vaginal length. Barring total vaginal length, all parameters are measured during maximal straining [2]. It is the only system that is recognized and approved by the three large relevant societies including the ICS, the American Urogynecology Society (AUGS), and the Society of Gynecology Surgeons for the quantification of pelvic organ prolapse, and the POPQ is arguably the most common quantification system in use by gynecologists [1].

In spite of POPQ having many merits to it and being a conventional system, POPQ has not succeeded to acquire an ecumenical importance since its use in routine clinical practice is still limited (40\% only) [3]. Many prodigious inconsistencies exist between the users of this staging system in terms of various aspects such as patient positioning, use or nonuse of a specula, and measurement of genital hiatus and perineal body at rest versus straining, perhaps indicating the complex nature and increase in the time consumption of POPQ, and thus being difficult to learn and incorporate into daily practice. Hence, this study was conducted with the aim of determining the association between the standard pelvic organ prolapsed quantification (POPQ) system and the simplified pelvic organ prolapsed (S-POP) quantification system.

\section{Materials and Methods}

This was a single-institution-based, prospective, and observational blinded study. A total of 100 patients with median age of $60(-/+10)$ years were studied, presenting at the GOPD of Safdarjung Hospital, New Delhi from July 2010 to June 2011. The Inclusion criteria for the patients were symptoms like something coming out of the vagina/ Urinary, or fecal incontinence/Pelvic fullness, or pressure/ Digital reposition of prolapsed part to urinate or defecate.

Prior to inclusion of any patient into this study, an informed consent was taken for the aforesaid purpose. Pelvic examinations were conducted by two groups of clinicians at each site, of which one by standard POPQ done by two clinicians ( 1 specialist +1 resident); and the second exam by S-POP by other two clinicians (1 specialist +1 resident). The order of such pelvic examinations was randomized, and examiners were blinded to each other's results. The examination was undertaken on patient after emptying the bladder and in lithotomy position and full valsalva maneuver (Fig. 1).

The above diagram depicts the 9 points of measurement in POPQ classification. The 4 points in red are measured only in POP classification.

For the S-POP, the four areas examined included the anterior and posterior vaginal walls, the apex, and the cervix. If a subject was status post-hysterectomy, then only three measurements were taken: the anterior and posterior vaginal walls, and the cuff scar/apex. No measuring devices are required for the S-POP, and the investigators use estimates for identifying those points on the anterior and posterior vaginal segments, which are used to represent the respective walls of the S-POP staging system for classification of each segment

Stage 1 Given point is $\geq 1 \mathrm{~cm}$ above hymen

Stage 2 Given point descends to the introitus, from $1 \mathrm{~cm}$ above to $1 \mathrm{~cm}$ below the hymen

Stage 3 Given point is $\geq 1 \mathrm{~cm}$ past hymen.

Stage 4 Complete vaginal vault eversion/procidentia.

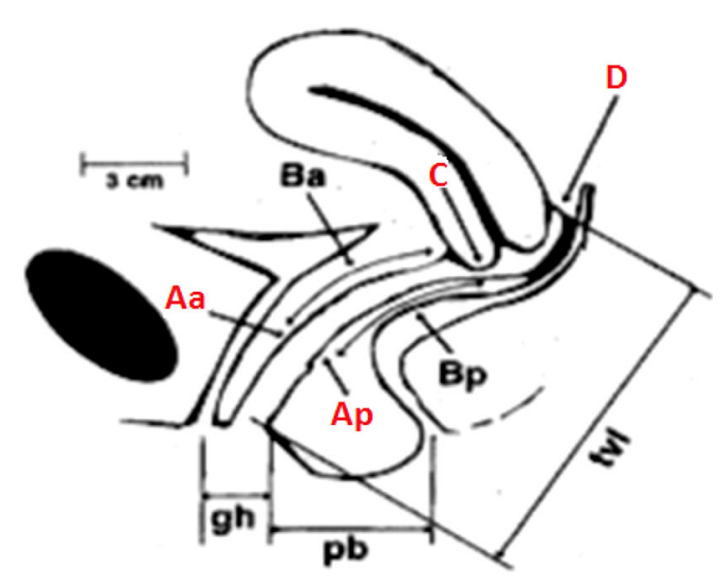

Fig. 1 Points of measurement for assessment of pelvic organ prolapse 


\section{Results}

One hundred patients with the average of 60 years $(+/-10)$ were included in this study, and the distribution among stages for the two different scoring systems is shown in table below:

The weighted kappa statistics for the intersystem reliability of the simplified prolapse classification system were 0.82 for the overall stage (Table 1 ); 0.83 and 0.86 for the anterior and posterior vaginal walls, respectively; and 0.89 and 0.81 for the cervix and posterior fornix, respectively. All these results demonstrate almost perfect agreement. Regarding kappa statistics, a value that lies between 0.81 and 1.0 is considered to indicate an almost perfect agreement beyond chance; if it lies between 0.61 and 0.80 , then it indicates a substantial agreement; if it lies between 0.41 and 0.60 , then it is considered to indicate a moderate agreement; and if it is below 0.40, then it becomes insignificant.

\section{Discussion}

The POPQ is a standard and precise examination system which is objective, site specific, and reproducible. The POPQ represented the collaboration of several international investigators and was adopted by the (International Continence Society (ICS), the American Urogynecologic Society (AUGS), and the (SGS) Society of Gynecologic Surgeons, establishing it as the first internationally

\begin{tabular}{lcc}
\hline Stage & POPQ $(\%)$ & S-POP (\%) \\
\hline I & 4 & 8 \\
II & 68 & 62 \\
III & 20 & 20 \\
IV & 8 & 10 \\
\hline
\end{tabular}

recognized POP classification system [4]. The POPQ system was also the first one to undergo extensive testing, and demonstrated excellent intra- and interexaminer reliability in four studies involving 240 subjects [6-9]. However, it has not gained any ecumenical status because of its limitations: it is less user friendly, is time consuming (2-3 min in expert hand), and demands expertise for its usage. Moreover, it does not offer any veridical statistical advantage over older simpler classification and its impact on surgical management. A survey of the members of ICS and AUGS, revealed that about one-third respondents did not use the POPQ system in routine practice [3]. The leading cause of this noncompliance was that it was too time consuming, as stated by almost one quarter of respondents. A sizeable number of respondents also found the system too confusing. Steele et al. [10], while assessing the ability to teach the POPQ to obstetrics and gynecology residents in 1998, found that only one of the 19 residents involved in this study stated they had used the system in the 3 months following the instruction and that only one resident knew a gynecologist (outside of the urogynecology division) who was using the POPQ.

The IUGA Standardization of Terminology Committee had developed a simplified version of the POPQ to address the problem of complexity and difficulty in the use of the POPQ system [4], and the feeling that a simplified system would be more applicable to daily clinical practice for the majority of healthcare providers, while the traditional POPQ should be followed for research purpose. The S-POP having approximate measurements and conspicuous classification pattern is quite user friendly as against the cumbersome nine-point exact measurements of the POPQ system. For swaying the implementation of S-POP, stage 0 of POPQ was omitted making it quite simple for generalized use. Stage 0 is not clinically significant. It may have relevance in research setting, but this is yet to be determined. The revised system is simpler in its format, approximates the measurement without the use of ruler,

Table 1 Intersystem reliability between the standard POPQ versus S-POP classification system

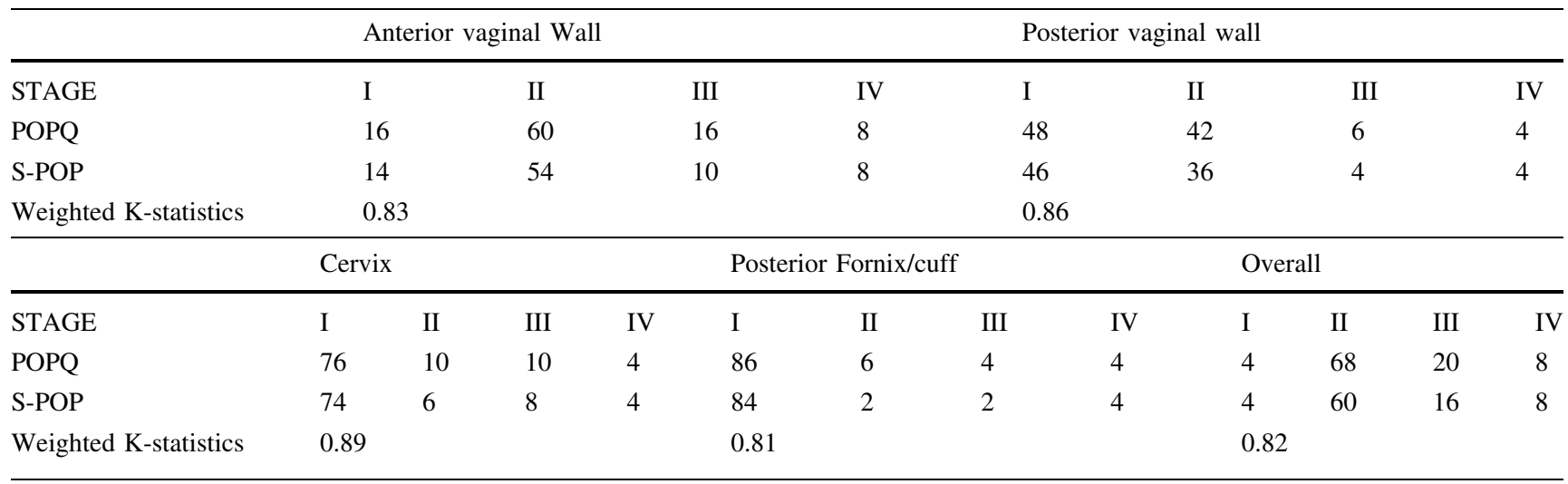


and less time $(<1 \mathrm{~min})$ is required for its execution with nominal expertise. It is similar to the Baden-Walker system of classification which had been used for many years.

In our study, according to the POPQ system, POP with stages $1,2,3$, and 4 were demonstrated in 4, 68, 20, and $8 \%$ subjects, respectively. The corresponding distribution patterns for respective stages were $8,62,20$, and $10 \%$ according to S-POP system. In $88 \%$ of the cases, the association between overall stages was identical, and it varied between systems by no more than one in any of the subjects. This was similar to the findings of the previous studies. The weighted kappa statistics for the intersystem reliability of the simplified prolapse classification system were 0.82 for the overall stage, indicating almost perfect association. This was comparable to the findings of Swift et al. [4] and Manonai et al. [5]. Our findings showed almost perfect association for each of the four subcategories, i.e., anterior and posterior vaginal wall, cervix, and posterior fornix, with values being $\geq 0.81$ in each category. This was comparable to the findings of Manonai et al. who showed only a substantial association for anterior and posterior vaginal wall subcategory. Swift et al., however, showed only a substantial association for posterior fornix/ apex subcategory.

Therefore, the results comparable to POPQ can be achieved with this system readily. However, its drawbacks remain: that it is not very site specific unlike POPQ. As in the POPQ staging system of prolapse, it too cannot be applied for complex prolapse, lateral vaginal wall defects, and high rectocele precluding its use for research purpose.

Ours is one of the first studies from the Indian subcontinent, to validate the usefulness of the simpler S-POP system in comparison with the more complex POPQ system. Larger multicenter trials, from within our country and globally, would help one establish the S-POP system as useful tool in the gynecologist's armamentarium, as well as spread awareness and increase popularity for its routine use in clinical practice, in comparison with the POPQ system.

\section{Conclusion}

There was appreciable intersystem association (reliability) between results of exams performed using the S-POP classification system and the POPQ system for classifying the stages of pelvic organ prolapse in a clinical population in this prospective, observational, and blinded study. Since the S-POP is less complicated and less time consuming than the POPQ and correlated well with the POP-Q, it would be more applicable to clinical practice for the majority of healthcare providers worldwide. However, for research purpose and for urogynecologists, POPQ will still remain the standard.

Conflict of interest None.

\section{References}

1. Kobashi KC. Evaluation of patients with urinary incontinence and pelvic prolapse. Campbell-Walsh textbook of Urology, 10th ed. Elsevier-Saunders; 2012.

2. Richter HE, Varner RE. Pelvic organ prolapse. Berek \& Novak's Gynecology, 14th ed. Lippincott Williams-Wilkins; 2007.

3. Auwad W, Freeman RM, Swift S. Is the pelvic organ prolapse quantification system (POPQ) being used? A survey of members of the International Continence Society (ICS) and the American Urogynecologic Society (AUGS). Int Urogynecol J. 2004;15: 324-7.

4. Swift S, Morris S, McKinnie V, et al. Validation of a simplified technique for using the POPQ pelvic organ prolapse classification system. Int Urogynecol J. 2006;17:615-20.

5. Manonai J, Mouritsen L, Palma P, et al. The inter-system association between the simplified pelvic organ prolapse quantification system (S-POP) and the standard pelvic organ prolapse quantification system (POPQ) in describing pelvic organ prolapsed. Int Urogynecol J. 2011;22:347-52.

6. Hall AF, Theofrastous JP, Cundiff GC, et al. Interobserver and intraobserver reliability of the proposed International Continence Society, Society of Gynecologic Surgeons, and American Urogynecologic Society pelvic organ prolapse classification system. Am J Obstet Gynecol. 1996;175:1467-71.

7. Athanasiou S, Hill S, Gleeson C, et al. Validation of the ICS proposed pelvic prolapse descriptive system. Neurourol Urodyn. 1995;14:414-5.

8. Schussler B, Peschers U. Standardisation of terminology of female genital prolapse according to the new ICS criteria: interexaminer reliability. Neurourol Urodyn. 1995;14:437-8.

9. Kobak WH, Rosenberger K, Walters MD. Interobserver variation in the assessment of pelvic organ prolapse. Int Urogynecol J Pelvic Floor Dysfunct. 1996;7:121-4.

10. Steele A, Mallapeddi P, Welgoss J, et al. Teaching the pelvic organ prolapse quantification system. Am J Obstet Gynecol. 1998;179:1458-64. 